\title{
Editorial
}

\section{Gaining an Accurate Diagnosis: A Multidisciplinary Approach}

\author{
Irina Shabalova \\ Department of Clinical Laboratory Diagnostics, Russian Medical Academy of Continuous Professional Education, \\ Moscow, Russia
}

The article by Gupta and Bhake [1] in this issue of Acta Cytologica is devoted to one of the most important problems in medicine: how to gain an accurate diagnosis. When we think about any disease from the point of view of diagnostics and treatment in the light of evidencebased medicine we usually use such terms as sensitivity, specificity, PPV, NPV, and others. We see that one method gives more informative results than another and decide to replace the second by the first. However, sometimes we forget that even if one person in 100,000 dies from a disease it can be a tragedy for his or her relatives. Being one of the great killers of the human race, tuberculosis still remains problematic from the point of view of detection and diagnosis; lymph node tuberculosis is twice as difficult for it is often lacking in typical symptoms such as cough and fever, as well as X-ray images, etc. Our experience has shown that in the past it was sometimes very difficult, if not impossible, to gain a correct diagnosis before surgery, and the image of specific granuloma was only revealed by rapid intraoperative cytology of the excised lymph node [2]. Nowadays, the possibility of using molecular methods opens new doors for morphologists, and from this point of view the theme of the study is highly important.

The design and methodology of scientific work allows us to analyze various methods, not per se but as part of a

\section{KARGER}

(C) 2017 S. Karger AG, Basel

E-Mail karger@karger.com

www.karger.com/acy complex. In the above-mentioned study [1], the obtained aspirates were divided into cytology (both Papanicolaou and Giemsa staining), PCR, and culture for analysis. Clinical data were also analyzed. Notwithstanding that PCR has good sensitivity compared to cytology, the results showed that there is no best diagnostic tool for all cases. Taking the histopathology and Ziehl-Neelsen staining as the gold standards, the authors demonstrated that there were cases negative for PCR and positive for ZiehlNeelsen staining, and vice versa. The results confirmed once more that when obtaining material from an enlarged lymph node we have to take tubercular lesion into account. Thus, taking material for morphology (MayGrünwald-Giemsa and Papanicolaou staining), PCR, and culture in the first visit can provide a multidisciplinary approach and an individual algorithm, and prompt accurate treatment of patients with enlarged lymph nodes.

\footnotetext{
References $\quad 1$ Gupta V, Bhake A: Assessment of clinically suspected tubercular lymphadenopathy by real-time PCR compared to non-molecular methods on lymph node aspirates. Acta Cytol 2017, DOI: $10.1159 / 000480064$.

2 Shabalova IP: Rapid cytology in lung, stomach and esophageal carcinoma intraoperative diagnosis. Probl Oncol 1985;7:727-732.
}

Correspondence to: Prof. Irina Shabalova Department of Laboratory Medicine Russian Medical Academy of Postgraduate Education 3rd Vladimirskaja 32/31, Apt. 129, RU-111397 Moscow (Russia) E-Mail irenshab@inbox.ru 